\title{
3D Simulation of Flexible Hypersonic Vehicles
}

\author{
Scott G. V. Frendreis* and Carlos E. S. Cesnik ${ }^{\dagger}$
}

\begin{abstract}
A framework for the analysis of flexible hypersonic vehicles in three-dimensional flight is discussed. Vehicle flexibility is modeled with a one-dimensional Euler-Bernoulli beam representation. The equations of motion are derived using a Lagrangian approach for the beam undergoing six rigid-body degrees of freedom flight. An unsteady aerodynamic model using steady shock-expansion theory with an unsteady correction from piston theory and a one-dimensional scramjet representation are used to determine the external loads on the vehicle. This model is then subjected to a stability analysis, including trimming the vehicle for a steady flight condition and linearizing its equations of motion about that trim state. The linearized system provides insight into the stability of the vehicle at a steady flight condition. Numerical results are given for both a rigid vehicle and one that is flexible, and the effect of flexibility on the stability properties of the vehicle is discussed.
\end{abstract}

\section{Introduction}

Maintaining controlled flight is among the greatest challenges facing the development of hypersonic vehicles. Complex interactions among the airframe, propulsion system, and vehicle aerodynamics prevent each vehicle subsystem from being developed independently. Because of this, computational flight simulations are a vital tool in the development of hypersonic vehicles.

Bolender and Doman ${ }^{1}$ have developed a two-dimensional flight dynamics vehicle code used to evaluate control algorithms, which was further extended by Oppenheimer et al. ${ }^{2}$ The model utilized 2D shock expansion theory, local piston theory, and Eckert's reference temperature method to calculate the vehicle aerodynamics. The scramjet was modeled as a 1D pipe with heat addition. This code has the capability to perform longitudinal flight simulations over a variety of vehicle trajectories in a computationally effective manner. However, the capability and accuracy of a two-dimensional model are inherently limited due to the fact that it is restricted to symmetric flight and cannot capture three-dimensional phenomena encountered in reality.

Recently, a similar simplified hypersonic vehicle flight dynamics simulation framework has been created for fully 3D flight. ${ }^{3}$ The approach coupled six-degree-of-freedom rigid body flight dynamics, steady aerodynamics (2D shock expansion and conical flow theories), and a simplified scramjet representation (1D pipe with heat addition). Using these individual submodels, the code first trims the vehicle for a steady flight condition and then linearizes the flight dynamics about the trimmed state. The 3D model was compared against the 2D model and was found to exhibit similar longitudinal flight dynamics. Additionally, the 3D model provided new insight into the lateral flight characteristics of the vehicle.

The current effort is to extend the 3D flight dynamics analysis framework to include the effects of vehicle flexibility and unsteady aerodynamics. The first step is to define the geometry of the vehicle and select a structural representation; for this study, a 1D beam representation is used. Then, the equations of motion are derived, capturing the coupling between the rigid body degrees of freedom and the structural degrees of freedom. An unsteady aerodynamic model is required, due to the inherently unsteady nature of flexible vehicle flight. The model used in this paper is a steady shock-expansion theory with an unsteady correction from piston theory. The existing 1D pipe flow with heat addition scramjet model is implemented to model the propulsion system. With the complete theoretical model, it is then possible to analyze the flight dynamics characteristics of the vehicle. First, it is trimmed for a steady cruise condition. Then, the equations of motion are linearized about the trim condition. The stability of the trim point is then characterized from the linear model. In order to demonstrate the effects of flexibility on the flight stability, the trim and linearization procedure is repeated for both rigid and flexible vehicle cases, and the results are compared.

\footnotetext{
*Graduate Research Assistant, University of Michigan, Email: scottgvf@umich.edu, Member AIAA.

${ }^{\dagger}$ Professor, Department of Aerospace Engineering, University of Michigan, Email: cesnik@umich.edu, 1320 Beal Avenue, 3024 FXB, Ann Arbor, MI 48109-2140, Ph. (734) 764-3397, Fax: (734) 764-0578, AIAA Associate Fellow.
} 


\section{Theoretical Model}

To analyze the flight characteristics of a flexible hypersonic vehicle, two components must be in place: the governing equations of motion and the capability to determine the external loads on the vehicle. The flexibility of the vehicle is captured using a 1D beam representation, and the aerodynamic model uses a 3D representation of the outer mold line (OML) of the vehicle. First, we set up the geometry and kinematics of the beam and the OML. Next, the equations of motion for a beam with six rigid-body degrees of freedom are derived using a Lagrangian approach. Then, the external loads coming from aerodynamics, propulsion, and gravity are expressed in terms of the flight conditions of the vehicle.

\section{A. Geometry and Kinematics}

The first step towards building the theoretical model is to determine the geometry and kinematics of the system. There are two components of the vehicle representation: the interior structure and the OML. The geometry of the interior structure is defined first, followed by the OML geometry. Then, the kinematic relations for the system are derived.

\section{Beam Geometry}

The flexibility of the vehicle is modeled as coming from an Euler-Bernoulli beam that has six rigid-body degrees of freedom. Consider a beam with a body-fixed frame of reference (labeled $B$ ) as shown in Fig. 1. This frame is comprised of the three orthogonal unit vectors, $\hat{e}_{B 1}, \hat{e}_{B 2}$, and $\hat{e}_{B 3}$, with associated coordinates $x_{1}, x_{2}$, and $x_{3}$. Note that in the remainder of this paper, $x$ with no subscript refers to the coordinate $x_{1}$. The origin of the $B$ frame is located on the elastic axis of the beam, and the elastic axis is aligned along $\hat{e}_{B 1}$. In addition, there is an inertial reference frame, labeled $E$, that is fixed to the Earth. The position of the origin of the $B$ frame with respect to the origin of the $E$ frame is $\vec{r}_{B / E}$. The orientation of the $B$ frame with respect to the $E$ frame is defined by the 3-2-1 Euler angles yaw $(\psi)$, pitch $(\theta)$, and roll $(\phi)$.

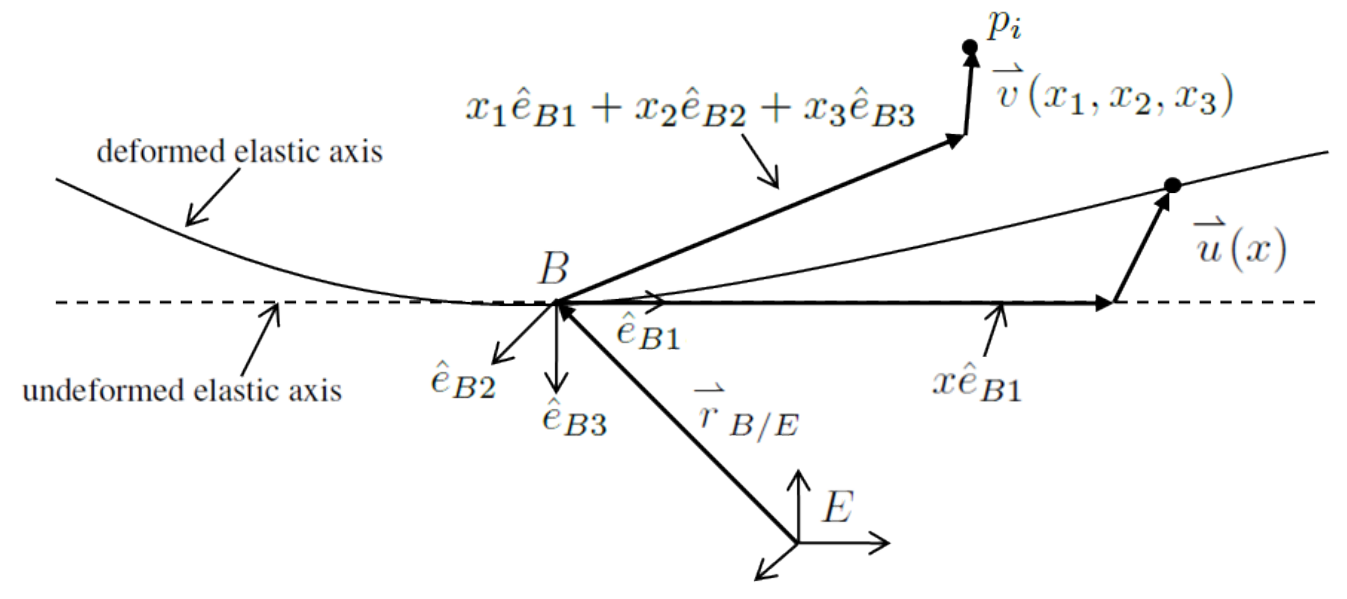

Figure 1. Geometry of flying beam system

At each point, $x$, along the beam axis, the beam can undergo up to four distinct deformations. These deformations are: extension along the $x_{1}$-direction $\left(u_{1}(x)\right)$, bending in the $x_{2}$-direction $\left(u_{2}(x)\right)$, bending in the $x_{3}$-direction $\left(u_{3}(x)\right)$ and torsion about the $x_{1}$ axis $(\theta(x))$. Each of these deformations is assumed to be small. The collective translational deformation of a point on the elastic axis, $\vec{u}(x)$, when expressed in the $B$ frame is

$$
\left.\vec{u}(x)\right|_{B}=\left\{\begin{array}{l}
u_{1}(x) \\
u_{2}(x) \\
u_{3}(x)
\end{array}\right\} .
$$

Thus, the location of a point on the beam elastic axis, $x$, with respect to the origin of the $E$ frame is

$$
\vec{r}_{x / E}=\vec{r}_{B / E}+x \hat{e}_{B 1}+\vec{u}(x)
$$


Additionally, there are several physical properties of the beam that are allowed to vary along its length, including:

- Mass/unit length, $\mu(x)$

- Rotational inertia about the $x$-axis/unit length, $I(x)$

- Extensional stiffness, $E A(x)$

- Bending stiffness, $E I_{z z}$ ( $x_{1}$-direction bending) and $E I_{y y}$ ( $x_{3}$-direction bending)

- Torsional stiffness, $G J(x)$

\section{2. $\quad$ Outer Mold Line Geometry}

The OML is a representation of the exterior of the vehicle that is used in determining the aerodynamic loads. Since the structure is able to deform, the OML must be able to deform as well; therefore, an OML consisting of a series of panels, as shown in Fig. 2, was chosen. Each panel is triangular, so that it will remain planar under arbitrary deformations. This specific OML geometry was provided by VSI ${ }^{\mathrm{a}}$ and is described in Ref. 4. The OML consists of

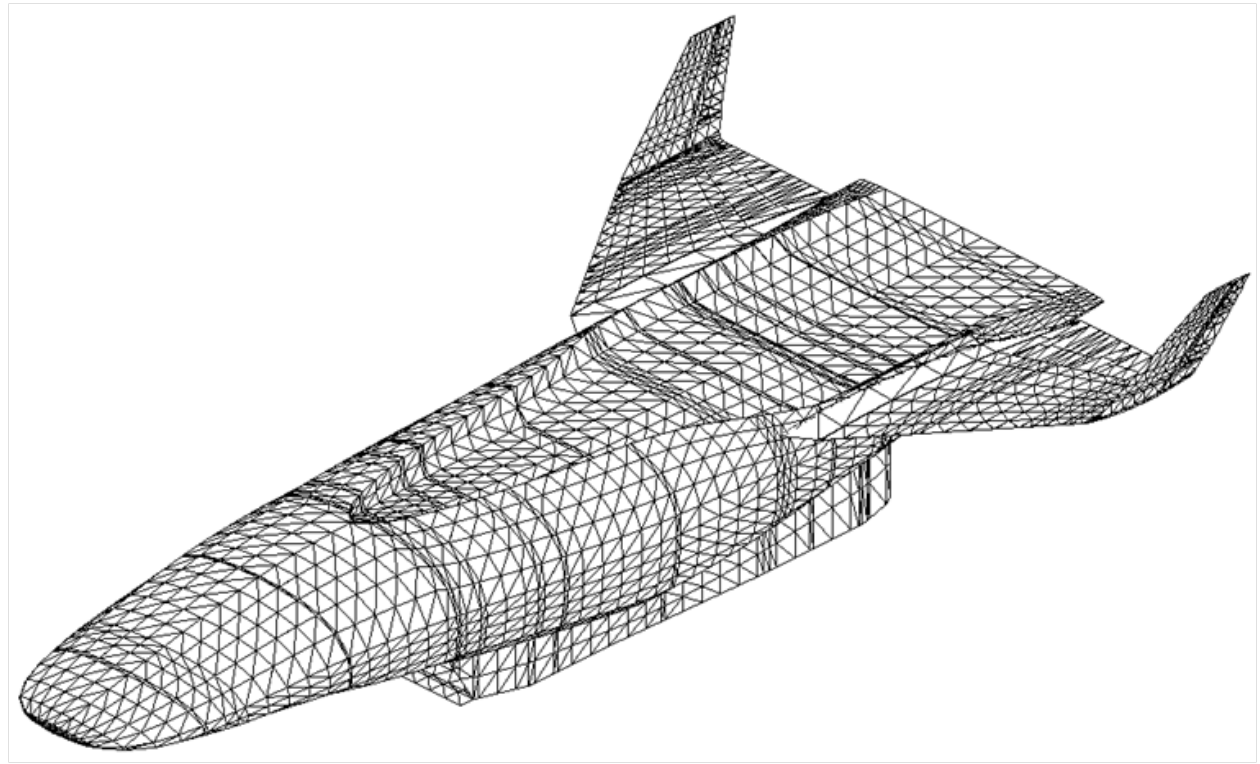

Figure 2. Isometric view of the outer mold line geometry.

a fuselage, scramjet cowl, and two stabilizers with elevator flaps. The recessed region on the top of the fuselage is where a spacecraft would be mounted when the hypersonic vehicle is being used for launch purposes.

There is a direct relation between the deformation of the OML and the deformation of the beam structural representation. Consider a point, $p_{i}$, located off of the beam axis at $\left(x_{1}, x_{2}, x_{3}\right)$ as shown in Fig. 1. This point can represent a location on the OML, such as the vertex of one of the triangular panels. In similar fashion to Eq. 2, the location of this point with respect to the $E$ frame can be expressed as

$$
\vec{r}_{p_{i} / E}=\vec{r}_{B / E}+x_{1} \hat{e}_{B 1}+x_{2} \hat{e}_{B 2}+x_{3} \hat{e}_{B 3}+\vec{v}\left(x_{1}, x_{2}, x_{3}\right),
$$

where $\vec{v}\left(x_{1}, x_{2}, x_{3}\right)$ is the change in position of $p_{i}$ due to internal structural deformations. It is assumed that each cross section of the undeformed OML taken normal to the undeformed elastic axis remains planar and normal to the elastic axis as it deforms. By imposing this assumption, the deformation vector, $\vec{v}\left(x_{1}, x_{2}, x_{3}\right)$, can be reduced to a function of $\vec{u}(x)$ and $\theta(x)$ through the relation

$$
\vec{v}\left(x_{1}, x_{2}, x_{3}\right)=\vec{u}\left(x_{1}\right)+\theta\left(x_{1}\right) \hat{e}_{B 1} \times\left(x_{2} \hat{e}_{B 2}+x_{3} \hat{e}_{B 3}\right)-\left(x_{2} u_{2}^{\prime}\left(x_{1}\right)+x_{3} u_{3}^{\prime}\left(x_{1}\right)\right) \hat{e}_{B 1}
$$

where $(\cdot)^{\prime}$ indicates the first derivative with respect to $x$.

${ }^{a}$ Vibroacoustics Solutions, Inc. 2214 229th Place Ames, Iowa 50014 


\section{Kinematics}

Before it is possible to calculate the total kinetic energy of the beam, it is necessary to determine the total velocity of each point on the beam with respect to the origin of the inertial reference frame. This is accomplished by taking the time derivative of Eq. 2 with respect to the $E$ frame. While it is simple to take the $E$ frame time derivative of the $\vec{r}_{B / E}$ term, it was found to be advantageous to take the $B$ frame time derivative of the $x \hat{e}_{B 1}$ and $\vec{u}(x)$ vectors. Since this involves taking the time derivative in a moving reference frame, it is necessary to introduce $\vec{\omega}_{B / E}$, the rotational velocity vector of the $B$ frame with respect to the $E$ frame. Using this definition, it is possible to calculate the $E$ frame time derivative of the position of a point, $x$, on the beam,

$$
\stackrel{E}{r}_{x / E}=\stackrel{E}{r}_{B / E}^{B}+x \hat{e}_{B 1}+\stackrel{B}{\vec{u}}(x)+\vec{\omega}_{B / E} \times\left(x \hat{e}_{B 1}+\vec{u}(x)\right) .
$$

Since $\hat{e}_{B 1}$ is constant in the $B$ frame, its $B$ frame time derivative, $\hat{e}_{B 1}^{B}$, is identically zero. Using this simplification, Eq. 5 can be reduced to

$$
\stackrel{E}{r}_{x / E}=\stackrel{E}{r}_{B / E}+\stackrel{B}{\vec{u}}(x)+\vec{\omega}_{B / E} \times\left(x \hat{e}_{B 1}+\vec{u}(x)\right) .
$$

Using a similar procedure on Eq. 3, the $E$ frame time derivative of a point, $p_{i}$, located off of the beam axis can be expressed as

$$
\stackrel{E .}{r}_{p_{i} / E}=\stackrel{E .}{r}_{B / E}+\stackrel{B .}{\vec{v}}\left(x_{1}, x_{2}, x_{3}\right)+\vec{\omega}_{B / E} \times\left(x_{1} \hat{e}_{B 1}+x_{2} \hat{e}_{B 2}+x_{3} \hat{e}_{B 3}+\vec{v}\left(x_{1}, x_{2}, x_{3}\right)\right) .
$$

\section{B. Equations of Motion}

The equations of motion for the flexible hypersonic vehicle in 3D flight are derived using a Lagrangian approach. This approach was chosen instead of a Newtonian approach because it avoids having to calculate internal forces in the structure. Using the geometric and kinematic relations developed in the previous section, we define the generalized coordinates and velocities of the system. Then, it is possible to compute the total kinetic and potential energies. From there, Lagrange's equations are applied to determine the equations of motion.

\section{Generalized Coordinates and Velocities}

Before assembling the total kinetic and potential energies of the system, it is necessary to express the velocity of a point on the beam, Eq. 6, in terms of generalized coordinates and velocities. It is assumed that a beam modal analysis is performed a priori and a set of mode shapes are retained for analysis. Each mode, $i$, has an associated, time-varying coordinate, $\eta_{i}$, and may consist of any combination of deformations (extension, bending, and torsion). Therefore, the structural deformations and velocities, $\vec{u}(x), \vec{u} \cdot \vec{u}(x), \theta(x)$, and $\dot{\theta}(x)$, are expressed in the $B$ frame as

$$
\begin{gathered}
\left.\vec{u}(x)\right|_{B}=\sum_{i=1}^{n} u_{i}(x) \eta_{i}=U(x) \eta, \\
\left.\vec{u}(x)\right|_{B}=\sum_{i=1}^{n} u_{i}(x) \dot{\eta}_{i}=U(x) \dot{\eta}, \\
\theta(x)=\sum_{i=1}^{n} \theta_{i}(x) \eta_{i}=\Theta(x) \eta, \\
\dot{\theta}(x)=\sum_{i=1}^{n} \theta_{i}(x) \dot{\eta}_{i}=\Theta(x) \dot{\eta},
\end{gathered}
$$

where $n$ is the number of structural modes, $U(x)$ is a 3-by-n matrix and $\Theta(x)$ is a 1-by- $n$ matrix. If we express $\stackrel{E}{r}_{B / E}$ and $\vec{\omega}_{B / E}$ in $B$ frame components, then we can define the following generalized velocities for the rigid body translational and rotational degrees of freedom, respectively,

$$
\left.\beta \equiv \stackrel{E}{r}_{B / E}\right|_{B}
$$




$$
\left.\zeta \equiv \vec{\omega}_{B / E}\right|_{B}
$$

Finally, noting that the undeformed position of a point on the elastic axis with respect to the $B$ frame origin is

$$
\left.x \hat{e}_{B 1}\right|_{B}=\left\{\begin{array}{l}
x \\
0 \\
0
\end{array}\right\}=x e_{1},
$$

where $e_{1} \equiv\left\lfloor\begin{array}{lll}1 & 0 & 0\end{array}\right]^{T}$. Eq. 6 can now be rewritten in terms of the generalized coordinates and velocities,

$$
\stackrel{E}{r}_{x /\left.E\right|_{B}}=\beta+U(x) \dot{\eta}+\tilde{\zeta}\left(x e_{1}+U(x) \eta\right),
$$

where

$$
\tilde{\zeta} \equiv\left[\begin{array}{ccc}
0 & -\zeta_{3} & \zeta_{2} \\
\zeta_{3} & 0 & -\zeta_{1} \\
-\zeta_{2} & \zeta_{1} & 0
\end{array}\right]
$$

\section{Total Kinetic Energy}

Consider an infinitesimal element of the beam in Fig. 1 at location $x$ along the span. The total kinetic energy of this element due to translation and rotation about the beam axis is

$$
T(x)=\frac{1}{2} \mu(x) \stackrel{E}{\stackrel{E}{r}_{x / E}} \cdot \stackrel{E}{r}_{x / E}+\frac{1}{2} I(x)\left(\zeta_{1}+\dot{\theta}(x)\right)^{2} .
$$

Using Eqs. 15 and 10, the kinetic energy of the beam element may be expressed in terms of the generalized coordinates and velocities,

$$
\begin{gathered}
T(x)=\frac{1}{2} \mu(x)\left(\beta+U(x) \dot{\eta}+\tilde{\zeta}\left(x e_{1}+U(x) \eta\right)\right)^{T}\left(\beta+U(x) \dot{\eta}+\tilde{\zeta}\left(x e_{1}+U(x) \eta\right)\right)+\ldots \\
\frac{1}{2} I(x)\left(\zeta_{1}+\Theta(x) \dot{\eta}\right)^{T}\left(\zeta_{1}+\Theta(x) \dot{\eta}\right)
\end{gathered}
$$

where $(\tilde{\cdot})$ is the skew-symmetric matrix operator. The total kinetic energy of the entire beam is obtained by integrating Eq. 2 with respect to $x$ along the span of the beam,

$$
\begin{gathered}
T=\frac{1}{2} \int \mu(x)\left(\beta+U(x) \dot{\eta}+\tilde{\zeta}\left(x e_{1}+U(x) \eta\right)\right)^{T}\left(\beta+U(x) \dot{\eta}+\tilde{\zeta}\left(x e_{1}+U(x) \eta\right)\right) d x+\ldots \\
\frac{1}{2} \int I(x)\left(\zeta_{1}+\Theta(x) \dot{\eta}\right)^{T}\left(\zeta_{1}+\Theta(x) \dot{\eta}\right) d x
\end{gathered}
$$

In this paper, all integrations are taken over the entire span of the beam, with respect to $x$; therefore, the limits of integration need not be explicitly stated for each integral. For this study, deformations are expected to be very small. Therefore, the $\tilde{\zeta} U(x) \eta$ terms in Eq. 2, which represent the change in inertia properties of the beam due to deformation, may be considered negligible. With this assumption, the final form of the total kinetic energy becomes

$$
T=\frac{1}{2} \int \mu(x)\left(\beta+U(x) \dot{\eta}+\tilde{\zeta} x e_{1}\right)^{T}\left(\beta+U(x) \dot{\eta}+\tilde{\zeta} x e_{1}\right) d x+\frac{1}{2} \int I(x)\left(\zeta_{1}+\Theta(x) \dot{\eta}\right)^{T}\left(\zeta_{1}+\Theta(x) \dot{\eta}\right) d x .
$$

\section{Total Potential Energy}

The Euler-Bernoulli beam undergoing 3D motion can experience four types of deformation: extension, torsion, and bending in two directions. Each of these deformations has an associated potential energy; the total potential energy due to these deformations is

$$
V=\frac{1}{2} \int\left(E A(x) u_{1}^{\prime 2}(x)+E I_{z z}(x) u_{2}^{\prime \prime 2}(x)+E I_{y y}(x) u_{3}^{\prime \prime 2}(x)+G J(x) \theta^{\prime 2}(x)\right) d x,
$$


where $(\cdot)^{\prime \prime}$ indicates the second derivative with respect to $x$. Using Eqs. 8 and 10, the total potential energy can be expressed in terms of the generalized structural coordinates,

$V=\frac{1}{2} \int \eta^{T}\left(U_{1}^{\prime T}(x) E A(x) U_{1}^{\prime}(x)+U_{2}^{\prime \prime T}(x) E I_{z z}(x) U_{2}^{\prime \prime}(x)+U_{3}^{\prime \prime T}(x) E I_{y y}(x) U_{3}^{\prime \prime}(x)+\Theta^{\prime T}(x) G J(x) \Theta^{\prime}(x)\right) \eta d x$,

where $U_{i}$ is the $i$ th row of the $U$ matrix.

\section{Application of Lagrange's Equations}

Once the total kinetic and potential energies are determined in terms of the generalized coordinates and velocities, Lagrange's equation can be applied. First, define the Lagrangian as $\mathcal{L} \equiv T-V$. Then, the equation of motion governing coordinate $i$ is

$$
\frac{d}{d t}\left(\frac{\partial \mathcal{L}}{\partial \dot{q}_{i}}\right)-\frac{\partial \mathcal{L}}{\partial q_{i}}=Q_{i},
$$

where $Q_{i}$ is the generalized force corresponding to coordinate $i$. When this is applied to the degrees of freedom corresponding to $\beta$, the resulting equations of motion are

$$
\int \mu(x)(\dot{\beta}+\tilde{\zeta} \beta) d x+\int x \mu(x)\left(T_{1} \dot{\zeta}+\tilde{\zeta} T_{1} \zeta\right) d x+\int \mu(x)(U(x) \ddot{\eta}+\tilde{\zeta} U(x) \dot{\eta}) d x=Q_{\beta}
$$

where $T_{1}$ is a constant matrix equal to $-\tilde{e_{1}}$. When Lagrange's equations are applied to the $\zeta$ degrees of freedom, the corresponding equations of motion are

$$
\begin{gathered}
\int x \mu(x) T_{1}^{T}(\dot{\beta}+\tilde{\zeta} \beta) d x+\int x^{2} \mu(x) T_{1}^{T}\left(T_{1} \dot{\zeta}+\tilde{\zeta} T_{1} \zeta\right) x+\int I(x) I_{1}^{T}\left(I_{1} \dot{\zeta}+\tilde{\zeta} I_{1} \zeta\right) x+\ldots \\
\int x \mu(x) T_{1}^{T}(U(x) \ddot{\eta}+\tilde{\zeta} U(x) \dot{\eta}) d x+\int I(x) I_{1}^{T}\left(e_{1} \Theta(x) \ddot{\eta}+\tilde{\zeta} e_{1} \Theta(x) \dot{\eta}\right) d x=Q_{\eta},
\end{gathered}
$$

where $I_{1}$ is a constant matrix equal to $e_{1} e_{1}^{T}$. Finally, Lagrange's equations are applied to the $\eta$ degrees of freedom, resulting in

$$
\begin{gathered}
\int \mu(x) U^{T}(x)(\dot{\beta}+\tilde{\zeta} \beta) d x+\int x \mu(x) U^{T}(x)\left(T_{1} \dot{\zeta}+\tilde{\zeta} T_{1} \zeta\right) d x+\int I(x) \Theta^{T}(x) e_{1}^{T}\left(I_{1} \dot{\zeta}+\tilde{\zeta} I_{1} \zeta\right) d x+\ldots \\
\int \mu(x) U^{T}(x)(U(x) \ddot{\eta}+\tilde{\zeta} U(x) \dot{\eta}) d x+\int I(x) \Theta^{T}(x) e_{1}^{T}\left(e_{1} \Theta(x) \ddot{\eta}+\tilde{\zeta} e_{1} \Theta(x) \dot{\eta}\right) d x+K \eta=Q_{\eta}
\end{gathered}
$$

where the stiffness matrix, $K$, is defined as

$$
K=\int\left(U_{1}^{\prime T}(x) E A(x) U_{1}^{\prime}(x)+U_{2}^{\prime \prime T}(x) E I_{z z}(x) U_{2}^{\prime \prime}(x)+U_{3}^{\prime \prime T}(x) E I_{y y}(x) U_{3}^{\prime \prime}(x)+\Theta^{\prime T}(x) G J(x) \Theta^{\prime}(x)\right) d x .
$$

Equations 24, 25, and 26 are the complete set of equations of motion for the flying beam system. It is convenient to assemble the three equations into a single matrix equation of the form

$$
M]\left\{\begin{array}{c}
\dot{\beta} \\
\dot{\zeta} \\
\ddot{\eta}
\end{array}\right\}+[C]\left\{\begin{array}{l}
\beta \\
\zeta \\
\dot{\eta}
\end{array}\right\}+\left\{\begin{array}{c}
0 \\
0 \\
K \eta
\end{array}\right\}=\left\{\begin{array}{c}
Q_{\beta} \\
Q_{\zeta} \\
Q_{\eta}
\end{array}\right\},
$$

where the mass matrix, $M$, is

$$
M=\left[\begin{array}{ccc}
\int \mu(x) d x & \int x \mu(x) T_{1} d x & \int \mu(x) U(x) d x \\
\int x \mu(x) T_{1}^{T} d x & \int x^{2} \mu(x) T_{1}^{T} T_{1} d x+\ldots & \int x \mu(x) T_{1}^{T} U(x) d x+\ldots \\
& \int I(x) I_{1}^{T} I_{1} d x & \int I(x) I_{1}^{T} e_{1} \Theta(x) d x \\
\int \mu(x) U^{T}(x) d x & \int x \mu(x) U^{T}(x) T_{1} d x+\ldots & \int \mu(x) U^{T}(x) U(x) d x+\ldots \\
& \int I(x) \Theta^{T}(x) e_{1}^{T} I_{1} d x & \int I(x) \Theta^{T}(x) \Theta(x) d x
\end{array}\right],
$$


and the damping matrix, $C$, is

$$
C=\left[\begin{array}{ccc}
\int \mu(x) \tilde{\zeta} d x & \int x \mu(x) \tilde{\zeta} T_{1} d x & \int \mu(x) \tilde{\zeta} U(x) d x \\
\int x \mu(x) T_{1}^{T} \tilde{\zeta} d x & \int x^{2} \mu(x) T_{1}^{T} \tilde{\zeta} T_{1} d x+\ldots & \int x \mu(x) T_{1}^{T} \tilde{\zeta} U(x) d x+\ldots \\
& \int I(x) I_{1}^{T} \tilde{\zeta} I_{1} d x & \int I(x) I_{1}^{T} \tilde{\zeta} e_{1} \Theta(x) d x \\
\int \mu(x) U^{T}(x) \tilde{\zeta} d x & \int x \mu(x) U^{T}(x) \tilde{\zeta} T_{1} d x+\ldots & \int \mu(x) U^{T}(x) \tilde{\zeta} U(x) d x+\ldots \\
& \int I(x) \Theta^{T}(x) e_{1}^{T} \tilde{\zeta} I_{1} d x & \int I(x) \Theta^{T}(x) e_{1}^{T} \tilde{\zeta} e_{1} \Theta(x) d x
\end{array}\right]
$$

The generalized forces, $Q_{\beta}, Q_{\zeta}$, and $Q_{\eta}$ are determined next.

\section{External Forces}

Once the equations of motion are assembled, the next task is to determine the generalized forces, $Q_{\beta}, Q_{\zeta}$, and $Q_{\eta}$. For a hypersonic vehicle, there are three sources of external forces: aerodynamics, propulsion, and gravity. For this study, the aerodynamic model consists of a combination of steady shock-expansion theory and unsteady piston theory. The propulsion model is a quasi-1D flow model with heat addition. The gravitational model assumes a uniform gravitational field that does not vary with altitude.

\section{Aerodynamic Forces}

The aerodynamic model determines the pressure distribution over the vehicle. Each panel that composes the exterior of the vehicle, as shown in Fig. 2, is assumed to have a uniform pressure distribution, which has both a steady and unsteady component. The full pressure distribution is solved on each panel independently with no flow information passed between panels.

The steady component of pressure is determined using shock-expansion theory. ${ }^{5}$ The two governing parameters in shock-expansion theory are the free stream Mach number, $M_{\infty}$, and the flow deflection angle, $\tau$. From these parameters, the pressure, temperature, density, and local speed of sound on the panel are determined. The unsteady pressure component is determined using a third-order unsteady pressure correction from piston theory. ${ }^{6}$ This correction uses local density, $\rho$, and speed of sound, $a$, calculated from the steady shock-expansion theory. The formula for the correction is

$$
\Delta p=\rho a^{2}\left(\frac{v_{n}}{a}+\frac{\gamma+1}{4}\left(\frac{v_{n}}{a}\right)^{2}+\frac{\gamma+1}{12}\left(\frac{v_{n}}{a}\right)^{3}\right),
$$

where $v_{n}$ is the normal component of the unsteady panel velocity and $\Delta p$ is the change in pressure.

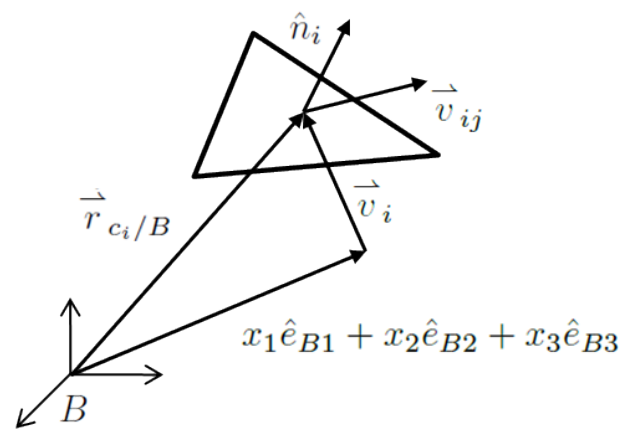

Figure 3. Outer mold line panel kinematics

Consider a single panel, $i$, located at $\left(x_{1}, x_{2}, x_{3}\right)$ as shown in Fig. 3, where $\vec{r}_{c_{i} / B}$ is the location of the centroid of the panel with respect to the $B$ frame origin and $\hat{n}_{i}$ is the unit normal vector. Define the quantity $\vec{v}_{i}=\vec{v}\left(x_{1}, x_{2}, x_{3}\right)$, which is the change in position of $c_{i}$ due to structural deformations, as defined in Eq. 4. As in Eq. 7, the total velocity of $c_{i}$ with respect to the origin of the $E$ frame can be written as

$$
\stackrel{E .}{r}_{c_{i} / E}=\stackrel{E}{r}_{B / E}+\stackrel{B .}{v}_{i}+\vec{\omega}_{B / E} \times\left(x_{1} \hat{e}_{B 1}+x_{2} \hat{e}_{B 2}+x_{3} \hat{e}_{B 3}+\vec{v}_{i}\right) .
$$


The position and velocity components due to structural deformations may be expressed in the $B$ frame as functions of the generalized structural coordinates and velocities as was done in Eqs. 8 and 9,

$$
\begin{aligned}
& \left.\vec{v}_{i}\right|_{B}=\left.\sum_{j=1}^{n} \vec{v}_{i j}\right|_{B} \eta_{j}=\sum_{j=1}^{n} v_{i j} \eta_{j}=V_{i} \eta, \\
& \left.\vec{v}_{i}\right|_{B}=\left.\sum_{j=1}^{n} \vec{v}_{i j}\right|_{B} \dot{\eta}_{j}=\sum_{j=1}^{n} v_{i j} \dot{\eta}_{j}=V_{i} \dot{\eta}
\end{aligned}
$$

Finally, the total velocity of $c_{i}$ can be expressed in the $B$ frame in terms of the generalized coordinates and velocities,

$$
\stackrel{E}{r}_{c_{i} /\left.E\right|_{B}}=\beta+V_{i} \dot{\eta}+\tilde{\zeta}\left(x_{1} e_{1}+x_{2} e_{2}+x_{3} e_{3}+V_{i} \eta\right)
$$

where $e_{2} \equiv\left\lfloor\begin{array}{lll}0 & 1 & 0\end{array}\right\rfloor^{T}$ and $e_{3} \equiv\left\lfloor\begin{array}{lll}0 & 0 & 1\end{array}\right\rfloor^{T}$. For a steady flight condition, the only nonzero term is $\beta$, which is referred to as the steady velocity component. The remaining terms in Eq. 33 are referred to as the unsteady velocity component.

For the steady aerodynamic analysis, the flow deflection angle, $\tau$, is determined from the panel normal vector and steady velocity component from

$$
\tau=\operatorname{acos}\left(\frac{n_{i}^{T} \beta}{\|\beta\|}\right)-\frac{\pi}{2},
$$

where $\left.n_{i} \equiv \hat{n}_{i}\right|_{B}$. The steady flow parameters on the panel are then determined from shock-expansion theory using the $\tau$. To apply the unsteady pressure correction from piston theory, the normal unsteady velocity component, $v_{n}$, is computed by the relation

$$
v_{n}=n_{i}^{T}\left(V_{i} \dot{\eta}+\tilde{\zeta}\left(x_{1} e_{1}+x_{2} e_{2}+x_{3} e_{3}+V_{i} \eta\right)\right)
$$

Once $v_{n}$ is computed, the unsteady correction is made, and the total panel pressure is obtained. This process is repeated for every panel on the OML.

The final step is determining the generalized forces due to the aerodynamic loads. The force on panel $i$ due to pressure, $p_{i}$, is

$$
\vec{f}_{i}=-p_{i} A_{i} \hat{n}_{i},
$$

where $A_{i}$ is the area of the panel. For $n$ panels, the net generalized forces due to aerodynamic loads are obtained by summing the individual contributions from each panel. The generalized force associated with $\beta$ is simply the sum of the panel forces expressed in the $B$ frame,

$$
Q_{\beta}=\left.\sum_{i=1}^{n} \vec{f}_{i}\right|_{B}
$$

The contribution of each panel force to $Q_{\zeta}$ is the moment it exerts about the origin of the $B$ frame,

$$
Q_{\zeta}=\left.\sum_{i=1}^{n}\left(\vec{r}_{c_{i} / B} \times \vec{f}_{i}\right)\right|_{B}
$$

To determine the contribution of each panel force to the generalized forces, $Q_{\eta}$, it is necessary to invoke the principle of virtual work. The virtual work due to a point force on panel $i$ associated with mode $j$ is

$$
\delta W_{j, i}=\vec{f}_{i} \cdot \delta\left(\vec{v}_{i j} \eta_{j}\right)=\left(\vec{f}_{i} \cdot \vec{v}_{i j}\right) \delta \eta_{j}=Q_{\eta j, i} \delta \eta_{j},
$$

where $Q_{\eta j, i}$ is the contribution of a point force on panel $i$ to generalized force $j$. The total generalized force associated with mode $j$ is obtained by summing the contributions from each panel,

$$
Q_{\eta_{j}}=\left.\sum_{i=1}^{n}\left(\vec{f}_{i} \cdot \vec{v}_{i j}\right)\right|_{B}
$$




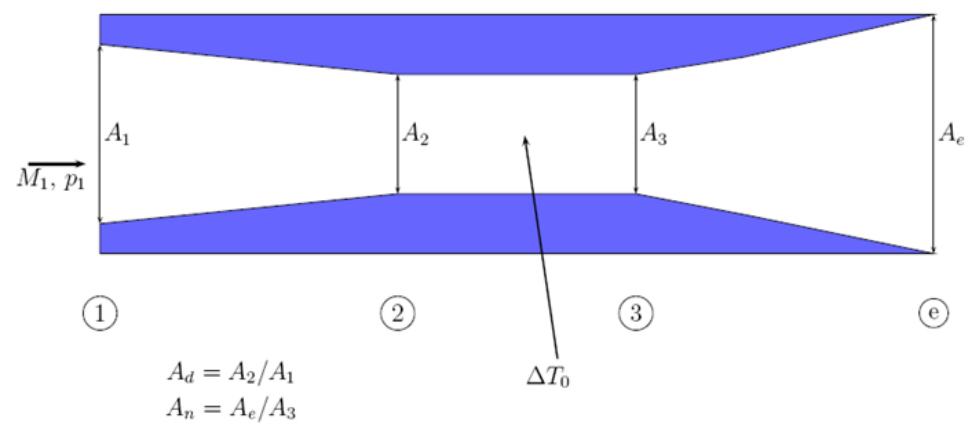

Figure 4. Scramjet flowpath diagram

\section{Propulsive Forces}

The scramjet propulsion model uses one-dimensional flow equations, as developed by Bolender and Doman. ${ }^{1}$ Note that, in the equations that follow, the subscripts refer to the zones shown in the schematic of the scramjet (Fig. 4).

It is assumed that the incoming air passes through a single oblique shock to align the flow with the engine. The first stage of the engine is an isentropic diffuser, which is represented by the isentropic mass continuity as

$$
\frac{\left[1+\frac{\gamma-1}{2} M_{2}^{2}\right]^{\frac{\gamma+1}{\gamma-1}}}{M_{2}^{2}}=A_{d}^{2} \frac{\left[1+\frac{\gamma-1}{2} M_{1}^{2}\right]^{\frac{\gamma+1}{\gamma-1}}}{M_{1}^{2}}
$$

where $M_{1}$ is the pre-diffuser Mach number, $M_{2}$ is the post-diffuser/pre-combustor Mach number, and $A_{d}$ is the diffuser area ratio. The combustor is treated as a constant area, frictionless duct with heat addition. The total temperature change in the combustor is governed by

$$
\frac{T_{03}}{T_{02}}=\frac{1+H_{f} \eta_{c} f_{s t} / c_{p} T_{02}}{1+f_{s t} \Phi}
$$

where $T_{02}$ and $T_{03}$ are the respective pre- and post-combustor total temperatures, $c_{p}$ is the specific heat capacity of air, $H_{f}$ is the lower heating value of the fuel, $\eta_{c}$ is the combustor efficiency, $f_{s t}$ is the stoichiometric fuel-to-air mass ratio, and $\Phi$ is the fuel equivalence ratio. Using the total temperature change, the post-combustor Mach number $M_{3}$ of the flow is given by

$$
\frac{M_{3}^{2}\left[1+\frac{\gamma-1}{2} M_{3}^{2}\right]}{\left(\gamma M_{3}^{2}+1\right)^{2}}=\frac{M_{2}^{2}\left(1+\frac{\gamma-1}{2} M_{2}^{2}\right)}{\left(\gamma M_{2}^{2}+1\right)^{2}}+\frac{M_{2}^{2}}{\left(\gamma M_{2}^{2}+1\right)^{2}} \frac{\left(T_{03}-T_{02}\right)}{T_{2}}
$$

Using the pre- and post-combustor Mach numbers, the post-combustor pressure and temperature are determined by the Rayleigh line relations

$$
\begin{gathered}
p_{3}=p_{2} \frac{1+\gamma M_{2}^{2}}{1+\gamma M_{3}^{2}} \\
T_{3}=\frac{M_{3}^{2}}{M_{2}^{2}} \frac{\left(1+\gamma M_{2}^{2}\right)^{2}}{\left(1+\gamma M_{1}^{2}\right)^{2}}
\end{gathered}
$$

where $p_{2}$ and $T_{2}$ are the pressure and temperature before combustion, and $p_{3}$ and $T_{3}$ are the pressure and temperature after combustion, respectively. The final stage of the engine is an isentropic supersonic nozzle. Since it is assumed to be isentropic, it is also governed by Eq. 41 . However, $M_{1}$ is replaced by $M_{3}, M_{2}$ is replaced by the engine exit Mach number $M_{e}$, and $A_{d}$ is replaced by the nozzle area ratio, $A_{n}$. Using momentum mechanics, the thrust force magnitude, $f_{T}$, is determined by

$$
f_{T}=\dot{m}_{a}\left[(1+\varepsilon) V_{e}-V_{\infty}\right]+\left(p_{e}-p_{\infty}\right) A_{e}-\left(p_{1}-p_{\infty}\right) A_{1}
$$

where $m_{a}$ is the engine inflow mass flow rate, $\varepsilon$ is the fuel-air ratio, $V_{e}$ is the flow's exit velocity, $V_{\infty}$ is the vehicle freestream velocity, $P_{e}$ is the exit pressure, $P_{\infty}$ is the freestream air pressure, $A_{e}$ is the engine exit area, and $A_{1}$ is the engine inlet area. 
The thrust is assumed to act along the $x$ axis of the $B$ frame, at an offset $d_{T}$ below the origin. Therefore, the contribution of thrust to $Q_{\beta}$ and $Q_{\zeta}$ is

$$
\begin{gathered}
Q_{\beta}=\left\{\begin{array}{c}
f_{T} \\
0 \\
0
\end{array}\right\} \\
Q_{\zeta}=\left\{\begin{array}{c}
0 \\
f_{T} d_{T} \\
0
\end{array}\right\} .
\end{gathered}
$$

While it is expected that the thrust force may make some contribution to $Q_{\eta}$, it is neglected for this study.

\section{Gravitational Forces}

The gravitational field is assumed to be uniform and invariant with respect to altitude. Since the $B$ frame origin is not necessarily located at the center of mass of the vehicle, the weight may exert both a force and moment. If $m$ is the total mass of the vehicle and $g$ is the gravitational constant, the contribution of weight to $Q_{\beta}$ is

$$
Q_{\beta}=m g\left\{\begin{array}{c}
-\sin (\theta) \\
\cos (\theta) \sin (\phi) \\
\cos (\theta) \cos (\phi)
\end{array}\right\} .
$$

If we further define the location of the center of mass with respect to the $B$ frame origin as $r_{c m}$, the contribution of weight to $Q_{\zeta}$ is

$$
Q_{\zeta}=m g \tilde{r}_{c m}\left\{\begin{array}{c}
-\sin (\theta) \\
\cos (\theta) \sin (\phi) \\
\cos (\theta) \cos (\phi)
\end{array}\right\} .
$$

As with the propulsive forces, the gravitational forces are assumed to have no effect on the generalized forces associated with the structural coordinates, $Q_{\eta}$.

\section{Flight Dynamics Analysis}

With the theoretical model completed, it is now possible to analyze the flight dynamics of the vehicle. The first stage of this analysis is to trim the vehicle for a steady cruise flight condition. The next stage is to characterize the stability of this trim condition by linearizing the equations of motion about that state. First, we discuss the theory behind these two processes. This is then followed by an example with numerical results.

\section{A. Trim}

The aircraft is trimmed for a steady-level flight condition. Steady-level flight implies that all accelerations $(\dot{\beta}, \dot{\zeta}$, and $\ddot{\eta})$, angular velocities $(\zeta)$, and structural velocities $(\dot{\eta})$ are identically zero. In addition, the roll angle of the aircraft, $\phi$, is zero. The translational velocity, $\beta$, and structural coordinates, $\eta$, may take on constant, nonzero values. When this set of conditions is applied to Eq. 28, the equations of motion reduce to

$$
\left\{\begin{array}{c}
Q_{\beta} \\
Q_{\zeta} \\
Q_{\eta}-K \eta
\end{array}\right\}=\left\{\begin{array}{l}
0 \\
0 \\
0
\end{array}\right\} .
$$

Any set of $\beta$ and $\eta$ that satisfy this equation are considered to be a valid trim condition. However, there are some additional constraints that are placed on the problem. Rather than allow all three components of $\beta$ to vary, the magnitude is held constant, and the angle of attack, $\alpha$, can vary. Then, $\beta$ takes the form

$$
\beta=\|\beta\|\left\{\begin{array}{c}
\cos (\alpha) \\
0 \\
\sin (\alpha)
\end{array}\right\} .
$$


In addition, the flight altitude and pitch angle must be constrained. For steady-level flight, the pitch angle, $\theta$, equals the angle of attack, $\alpha$.

In the process of satisfying Eq. 51, several states and control inputs are allowed to take on arbitrary values (with certain bounds). As already mentioned, the angle of attack may vary, as well as the structural coordinates, $\eta$. The two available control inputs are the elevator deflection angle, $\delta_{e}$, and the scramjet fuel equivalence ratio, $\Phi$.

For this study, the actual trimming of the vehicle is carried out numerically in Matlab using the fmincon function. Fmincon performs constrained minimization problems for a scalar-valued objective function. Since the goal is to satisfy Eq. 51, the objective function must take on its lowest value when this is satisfied. One such objective function is

$$
J=\left\lfloor\begin{array}{lll}
Q_{\beta} & Q_{\zeta} & Q_{\eta}-K \eta
\end{array}\right]\left[\begin{array}{c}
W \\
\end{array}\right]\left\{\begin{array}{c}
Q_{\beta} \\
Q_{\zeta} \\
Q_{\eta}-K \eta
\end{array}\right\},
$$

where $W$ is a weighting matrix used to scale the different generalized forces. This function evaluates to zero at the trimmed condition and is positive for all other conditions. The trimmed state is the set of input variables $\left(\alpha, \eta, \delta_{e}\right.$, and $\Phi)$ that bring $J$ to zero.

\section{B. Stability Analysis}

Once the trimmed state is reached, the next step is to evaluate the stability of that state. This is accomplished by linearizing the nonlinear equations of motion. The linearized equations of motion are put in state space form,

$$
\dot{x}=A x+B u
$$

where $x$ is the column vector of state perturbations from trim, $u$ is the vector of control input perturbations from trim, and $A$ and $B$ are constant matrices. The state perturbation vector is $x=\left[\begin{array}{lllllll}\beta & \zeta & \dot{\eta} & \eta & h & \phi & \theta\end{array}\right]^{T}$, and the control input perturbation vector is $u=\left\lfloor\begin{array}{ll}\delta_{e} & \Phi\end{array}\right\rfloor^{T}$.

The actual determination of the constant matrices $A$ and $B$ is done numerically in Simulink using the linmod tool. Using a file defining the full nonlinear equations of motion, linmod extracts the linear system about the specified trim state.

Once the linearized system is obtained, the stability of the open-loop system can be determined by examining the eigenvalues of the $A$ matrix. The eigenvalues may be real, imaginary, or complex. Real or complex eigenvalues with negative real parts indicate stable modes, while positive real parts indicate unstable modes. Purely imaginary eigenvalues represent a neutrally-stable condition. The shapes of the modes corresponding to each of the eigenvalues are given by their associated eigenvector.

\section{Numerical Results}

Numerical results are now presented to illustrate the flight dynamics analysis process just described. In order to highlight the effects of flexibility on the flight characteristics of a hypersonic vehicle, the trim and linearization procedure are performed on both a rigid and flexible vehicle. It is first necessary to select a set of numerical values for the geometric, inertia, and stiffness characteristics of the vehicle, as well as the beam mode shapes. The $B$ reference frame origin is fixed at the midpoint of the vehicle. The part of the vehicle to the front of the $B$ frame origin (i.e., with positive $x$ coordinate values) is referred to as the forebody; the region behind the $B$ frame origin is called the aftbody.

The vehicle parameters used for the flight dynamics analysis are presented in Table 1. The mass and stiffness properties were obtained by scaling the parameters used in Ref. 1 to the width of the vehicle. In order to maintain simplicity, three beam mode shapes are retained for the numerical results. The first mode shape consists of purely lateral bending $\left(u_{2}(x)\right)$, and the second mode shape consists of purely longitudinal bending $\left(u_{3}(x)\right)$. The final mode shape consists of torsion $(\theta(x))$ only. Therefore, the first mode for free-free beam-bending vibrations is used for both the first and second mode shapes. Bolender and Doman ${ }^{1}$ suggest modeling the beam as a set of two cantilevered beams connected at the origin of the $B$ frame. This maintains the required condition that the deflection and slope of the beam remain zero at the origin, since the $x$ axis is assumed to lie along the beam elastic axis. Setting $\lambda^{4} \equiv \omega^{2} \mu(x) / E I$, where $\omega$ is the unknown frequency of free vibration and $E I$ is assumed to be constant, the bending mode shape is of the form

$$
u_{2 i}(x)=A_{i} \sin (\lambda x)+B_{i} \cos (\lambda x)+C_{i} \sinh (\lambda x)+D_{i} \cosh (\lambda x),
$$


Table 1. Vehicle parameters

\begin{tabular}{ccc}
\hline \hline Parameter & Name & Value \\
\hline$L_{f}$ & Forebody length & $23.1 \mathrm{~m}$ \\
$L_{a}$ & Aftbody length & $23.1 \mathrm{~m}$ \\
$\mu_{f}$ & Forebody mass/unit length & $3.86 \times 10^{3} \mathrm{~kg} / \mathrm{m}$ \\
$\mu_{a}$ & Aftbody mass/unit length & $5.76 \times 10^{3} \mathrm{~kg} / \mathrm{m}$ \\
$I$ & Rotational inertia about $x$-axis/unit length & $7.41 \times 10^{5} \mathrm{kgm}^{2} / \mathrm{m}$ \\
$E I_{z z}$ & $x_{1}-x_{2}$ plane bending stiffness & $6.32 \times 10^{9} \mathrm{Nm}^{2}$ \\
$E I_{y y}$ & $x_{1}-x_{3}$ plane bending stiffness & $6.32 \times 10^{9} \mathrm{Nm}^{2}$ \\
$G J$ & Torsional stiffness & $4.74 \times 10^{9} \mathrm{Nm}^{2}$ \\
\hline \hline
\end{tabular}

where $i$ can refer to either the forebody $(f)$ or the aftbody $(a)$ and $A_{i}, B_{i}, C_{i}$, and $D_{i}$ are constants to be determined. The constants and $\lambda$ are determined by applying the boundary conditions for a cantilevered beam:

$$
\begin{aligned}
u_{2 f}(0) & =0 & u_{2 a}(0) & =0 \\
u_{2 f}^{\prime}(0) & =0 & u_{2 a}^{\prime}(0) & =0 \\
u_{2 f}^{\prime \prime}\left(L_{f}\right) & =0 & u_{2 a}^{\prime \prime}\left(-L_{a}\right) & =0 \\
u_{2 f}^{\prime \prime \prime}\left(L_{f}\right) & =0 & u_{2 a}^{\prime \prime \prime}\left(-L_{a}\right) & =0
\end{aligned}
$$

There are multiple values of $\lambda$ that satisfy these boundary conditions; the value corresponding to the first mode (i.e., to the lowest frequency) is selected. This gives the analytical expression for the first free-free bending mode shape,

$$
\begin{gathered}
u_{2 f}(x)=-0.367 \sin (-0.081 x)-0.5 \cos (-0.081 x)+0.367 \sinh (-0.081 x)+0.5 \cosh (-0.081 x), \\
u_{2 a}(x)=0.367 \sin (-0.081 x)-0.5 \cos (-0.081 x)-0.367 \sinh (-0.081 x)+0.5 \cosh (-0.081 x) .
\end{gathered}
$$

The beam displacement and rotation for this mode shape are shown in Figure 5. This mode shape is applied to both the first mode (lateral bending) and second mode (longitudinal bending).

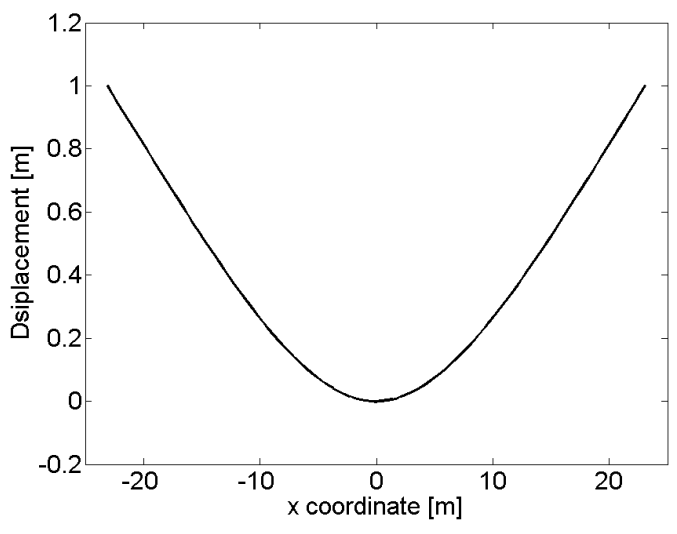

a) Beam displacement

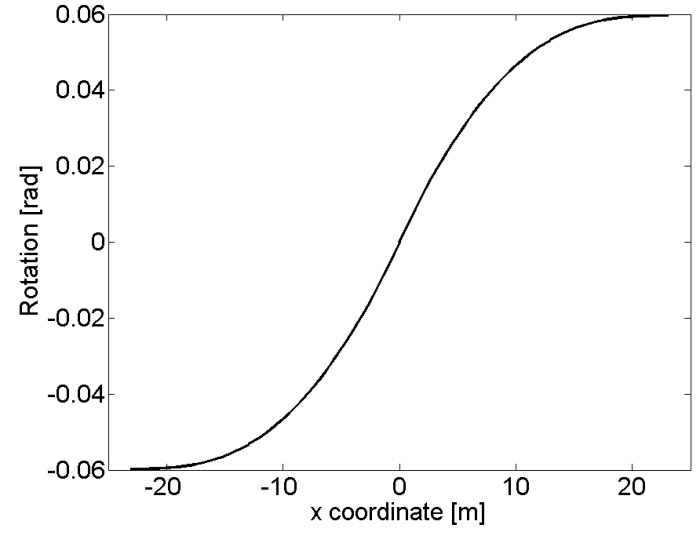

b) Beam rotation

Figure 5. First free-free beam-bending mode shape

Similarly, the first mode for free-free torsional vibrations is used for the third mode shape. Again, this mode shape is obtained by assuming the two help members are clamped together at the origin of the $B$ frame. The analytical expression for the torsional mode shape is

$$
\theta_{f}(x)=\sin \left(\frac{\pi x}{2 L_{f}}\right),
$$




$$
\theta_{a}(x)=\sin \left(\frac{\pi x}{2 L_{a}}\right) .
$$

With all necessary parameter values selected and mode shapes defined, the next step is to determine a trim condition for both the rigid-body and flexible cases. The vehicle is trimmed for Mach 8, steady-level flight at an altitude of $26 \mathrm{~km}$. In trimming the rigid vehicle, the flexible states, $\eta$, are all constrained to be zero. The trimmed values of angle of attack, structural modal displacements, elevator deflection, and scramjet fuel-equivalence ratio are presented in Table 2 for both the rigid-body and flexible cases. Note that the negative amplitude of the longitudinal bending modal displacement represents the nose and tail deflecting upward, due to the orientation of the $B$ frame.

Table 2. Trimmed state

\begin{tabular}{cccc}
\hline \hline Parameter & Name & Value (rigid-body) & Value (flexible) \\
\hline$\alpha$ & Angle of attack & $2.94 \mathrm{deg}$ & $2.54 \mathrm{deg}$ \\
$\eta_{1}$ & Modal displacement, lateral bending & - & 0 \\
$\eta_{2}$ & Modal displacement, longitudinal bending & - & -0.171 \\
$\eta_{3}$ & Modal rotation, torsion & - & 0 \\
$\delta_{e}$ & Elevator deflection angle & $1.65 \mathrm{deg}$ & $-8.16 \mathrm{deg}$ \\
$\Phi$ & Scramjet fuel-equivalence ratio & 0.442 & 0.435 \\
\hline \hline
\end{tabular}

Finally, the governing equations of motion are linearized about the trim conditions and put in state space form, as in Eq. 54. The eigenvalues of the $A$ matrix are presented in Table 3 for the rigid-body case and Table 4 for the flexible case. Damping ratio, damped natural frequency, and mode names are also given for each mode. Note that several

Table 3. Eigenvalues of Linearized 3D System, Rigid-Body Case

\begin{tabular}{cccc}
\hline \hline Eigenvalue & Damping Ratio & Damped Natural Frequency (rad/s) & Mode \\
\hline-2.08 & 1.00 & 0 & Short Period \\
1.92 & -1.00 & 0 & Short Period \\
$-1.21 \times 10^{-6} \pm 3.92 \times 10^{-2} j$ & $3.08 \times 10^{-5}$ & $3.92 \times 10^{-2}$ & Phugoid-Altitude \\
$-5.09 \times 10^{-4}$ & 1.00 & 0 & Phugoid-Altitude \\
$-1.47 \times 10^{-2} \pm 0.181 j$ & $8.08 \times 10^{-2}$ & 0.181 & Dutch Roll \\
$-8.43 \times 10^{-3} \pm 1.46 \times 10^{-2} j$ & 0.499 & $1.46 \times 10^{-2}$ & Roll-Spiral \\
\hline \hline
\end{tabular}

Table 4. Eigenvalues of Linearized 3D System, Flexible Case

\begin{tabular}{cccc}
\hline \hline Eigenvalue & Damping Ratio & Damped Natural Frequency (rad/s) & Mode \\
\hline-2.05 & 1.00 & 0 & Short Period \\
1.88 & -1.00 & 0 & Short Period \\
$-2.21 \times 10^{-5} \pm 4.36 \times 10^{-2} j$ & $5.07 \times 10^{-4}$ & $4.36 \times 10^{-2}$ & Phugoid-Altitude \\
$-6.01 \times 10^{-4}$ & 1.00 & 0 & Phugoid-Altitude \\
$-0.109 \pm 0.250 j$ & 0.399 & 0.250 & Dutch Roll \\
$8.57 \times 10^{-2} \pm 0.238 j$ & -0.339 & 0.238 & Roll-Spiral \\
$-9.84 \times 10^{-3} \pm 12.3 j$ & $8.01 \times 10^{-4}$ & 12.3 & Lateral Bending \\
$-3.39 \times 10^{-2} \pm 12.1 j$ & $2.80 \times 10^{-3}$ & 12.1 & Longitudinal Bending \\
$-7.77 \times 10^{-3} \pm 5.53 j$ & $1.40 \times 10^{-3}$ & 5.53 & Torsion \\
\hline \hline
\end{tabular}


of the mode names match those of classical rigid-body flight modes ${ }^{7}$ (phugoid, Dutch roll, etc.). These names were assigned to the hypersonic vehicle mode that best matched the classical mode. However, some of these hypersonic vehicle modes exhibit very different behavior from their classical counterpart.

The first set of eigenvalues correspond to the short-period mode. The classical short period mode is a steady, welldamped oscillation dominated by angle of attack and pitch rate. For the hypersonic vehicle, the short-period mode is found to exhibit an unstable, exponential behavior in both the rigid and flexible cases. The classical phugoid mode is a lightly-damped, stable oscillation in flight speed and altitude. Due to the inclusion of altitude as a state variable, the hypersonic vehicle phugoid mode (referred to as the phugoid-altitude mode) is a stable third-order mode with two complex eigenvalues and one real eigenvalue. The classical Dutch-roll mode is a stable oscillation between sideslip and yaw rate. In both the rigid-body and flexible cases for the hypersonic vehicle, the classical Dutch-roll behavior is observed. The final mode observed in both the rigid-body and flexible models is the roll-spiral mode. This corresponds to an oscillatory motion dominated by roll angle, roll rate, sideslip, and yaw rate. This mode is particularly interesting, because it is stable for the rigid-body case and unstable for the flexible case. The final three modes for the flexible hypersonic vehicle are primarily structural modes, all three of which are stable and oscillatory.

A striking result from this analysis is that one of the flight modes (the roll-spiral mode) becomes unstable when vehicle flexibility is included. To investigate how the stability properties are impacted by flexibility, the trim and linearization procedure are performed for incrementally increasing values of structural stiffness. All three stiffness values $\left(E I_{z z}, E I_{y y}\right.$, and $\left.G J\right)$ are multiplied by a scaling factor, $\sigma$, which effectively increases the overall stiffness of the vehicle. The values of $\sigma$ used for this study are 1,2,10,100, and 1000, where $\sigma=1$ represents the original set of stiffness properties listed in Table 1. The trimmed values of $\alpha, \eta_{2}, \delta_{e}$, and $\Phi$ for varying $\sigma$ are shown in Fig. 6 .
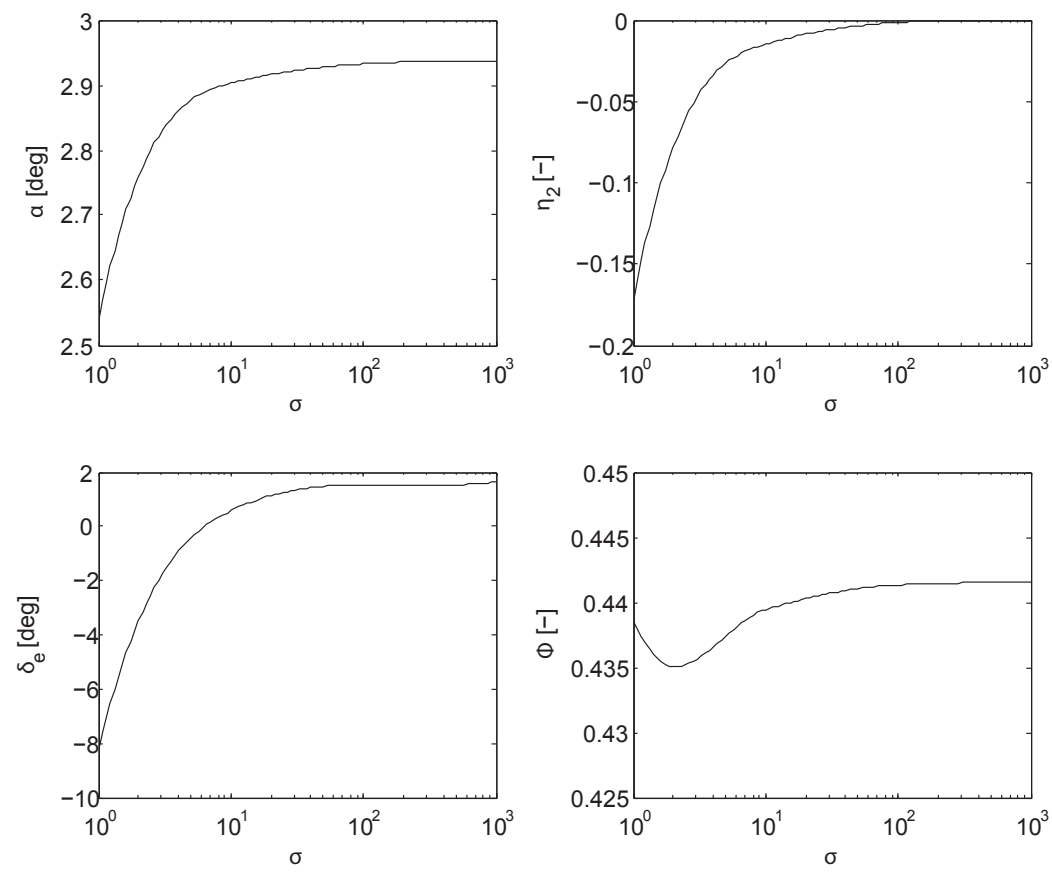

Figure 6. Trimmed states and control inputs for varying $\sigma$ values

The system is then linearized about the trim state for each value of $\sigma$. To see how the eigenvalues vary with increasing stiffness, they are displayed in a root-locus plot, Fig. 7, along with the eigenvalues corresponding to a rigid vehicle (as listed in Table 3). The plot is zoomed-in to show how the rigid-body mode eigenvalues vary with stiffness. The structural mode eigenvalues are not shown because increasing $\sigma$ values simply increase their frequency without impacting their stability. Also, the short-period eigenvalues are not shown because they are not substantially altered by flexibility. The first thing to note is that each eigenvalue approaches its corresponding rigid-body value as stiffness is increased. Whereas the phugoid-altitude mode eigenvalues remain near their rigid-body values for all levels of flexibility, the Dutch roll and roll-spiral mode eigenvalues vary substantially with flexibility. The Dutch roll mode eigenvalues are stable for all cases, but their damping decreases at higher stiffness values. The roll-spiral mode is unstable for the most flexible cases but transitions to stable between $\sigma$ values of 10 and 100. Since all stiffness 


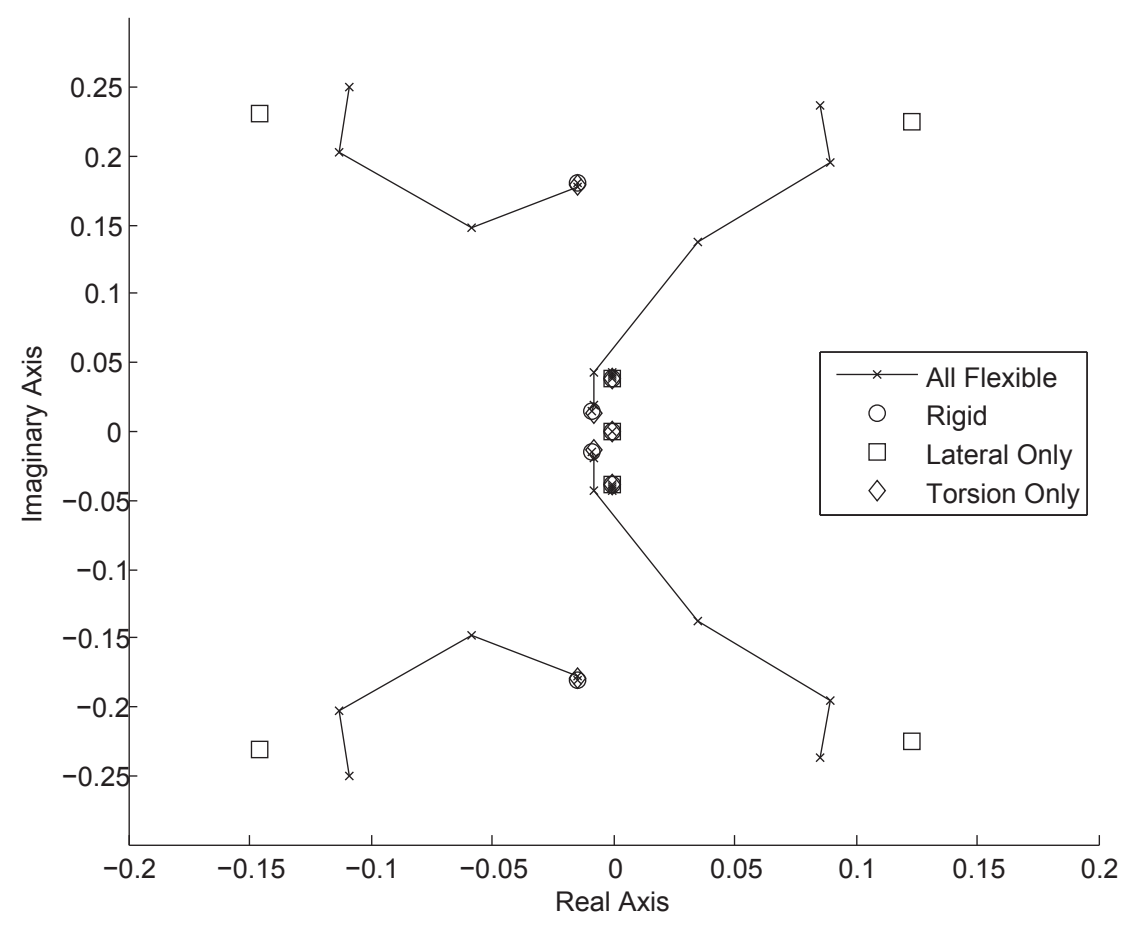

Figure 7. Root-locus of rigid-body mode eigenvalues for varying $\sigma$ values

properties are increased proportionally, it is unclear if flexibility in a single structural mode is causing the significant change in Dutch roll and roll-spiral mode behavior, or if it is a combined effect from multiple structural modes. To address this question, two additional cases are analyzed; in each case, only one structural mode is included with the other two constrained to be rigid. The first case includes lateral bending only, and the second case includes pure torsion. In both cases, the original stiffness value given in Table 1 is used (i.e., $\sigma=1$ ). The eigenvalues for both cases are shown in Fig. 7. For lateral bending only, the spiral-roll mode is unstable, and both the Dutch roll and rollspiral eigenvalues are near their corresponding eigenvalues for the original fully-flexible vehicle. When only torsion is included, all of the eigenvalues are near their rigid-body values. Therefore, it can be concluded that flexibility in the lateral bending mode is having the most influence on the change in behavior of the lateral flight modes.

While these results only represent the flight characteristics at one flight condition for a limited set of stiffness properties, they demonstrate the capabilities of this type of analysis. Some flight modes are affected minimally by the inclusion of flexibility, such as the short-period mode, while others are altered significantly. In the case of the roll-spiral mode, insufficient lateral bending stiffness induces instability in an otherwise stable flight mode.

\section{Conclusions}

This paper describes a three-dimensional flexible hypersonic vehicle simulation framework for the design and evaluation of flight control systems, as well as analysis of flight stability. In this implementation, a beam representation is used to model the structural flexibility of the aircraft. The coupled rigid body-structural equations of motion are derived using a Lagrangian approach. An outer mold line geometry consisting of deformable panels was obtained to determine the aerodynamic loads. The aerodynamic model is applied to each panel independently and uses a combination of steady shock-expansion theory and an unsteady pressure correction from piston theory. The propulsion system is modeled as quasi one-dimensional flow with heat addition.

The vehicle is trimmed for a steady-level flight condition, and its governing equations of motion are linearized about the trim state. This process is performed for both rigid-body and flexible cases. The eigenvalues of the linearized system are then used to characterize the stability of the trim point. For the set of parameters used in this study, it is determined that the inclusion of flexibility alters the stability properties of the rigid-body flight modes. Future additions to this model include a more accurate two dimensional scramjet model, an improved unsteady aerodynamic model with viscosity and shock interactions, and the effects of thermoelasticity. 


\section{Acknowledgements}

This research is funded by NASA award NNX08AB32A (Don Soloway and Jorge Bardina as program managers ) and by the Air Force Research Laboratory/Air Vehicles Directorate grant FA 8650-07-2-3744 for the Michigan/AFRL Collaborative Center in Control Sciences (Michael Bolender as technical monitor).

\section{References}

\footnotetext{
${ }^{1}$ Bolender, M. A. and Doman, D. B., "Nonlinear Longitudinal Dynamical Model of an Air-Breathing Hypersonic Vehicle," Journal of Spacecraft and Rockets, Vol. 44, No. 2, 2007, pp. 374-387.

${ }^{2}$ Oppenheimer, M. W., Skujins, T., Bolender, M. A., and Doman, D. B., "A Flexible Hypersonic Vehicle Model Developed with Piston Theory," Proceedings of the 2007 Atmospheric Flight Mechanics Conference and Exhibit, AIAA Paper No. 2007-6396, August 2007.

${ }^{3}$ Frendreis, S. G. V., Skujins, T., and Cesnik, C. E. S., "Six-Degree-of-Freedom Simulation of Hypersonic Vehicles," AIAA Atmospheric Flight Mechanics Conference, 2009, AIAA Paper 2009-5601.

${ }^{4}$ Vogel, J. M., Kelkar, A. G., Inger, G., Whitmer, C., Sidlinger, A., and Rodriguez, A., "Control-Relevant Modeling of Hypersonic Vehicles," 2009 American Control Conference, 2009.

${ }^{5}$ Anderson, J. D., Modern Compressible Flow with Historical Perspective, McGraw-Hill, 2002.

${ }^{6}$ McNamara, J. J. and Friedmann, P. P., "Aeroelastic and Aerothermoelastic Analysis of Hypersonic Vehicles: Current Status and Future Trends," Proceedings of the 48th AIAA/ASME/ASCE/AHS/ASC Structures, Structural Dynamics, and Materials Conference, 2007.

${ }^{7}$ Stengel, R. F., Flight Dynamics, Princeton University Press, Princeton, NJ, 2004.
} 